\title{
Passive Gain-Equalized Wide-Band Erbium-Doped Fiber Amplifier Using Samarium-Doped Fiber
}

\author{
Shien-Kuei Liaw and Yung-Kuang Chen
}

\begin{abstract}
A passive gain-equalization technique of erbiumdoped fiber amplifier (EDFA) using a samarium-doped fiber to obtain wide-band flattened gain operation is reported. In the wavelength range from 1529-1559-nm, small inter-channel gain variations can be achieved. This simple technique is suitable for multiwavelength wide-band in-line-amplifier cascaded transmission applications.
\end{abstract}

\section{INTRODUCTION}

G AIN-EQUALIZATION is one of the major problems facing multiple-channel wavelength-division-multiplexing (WDM) lightwave systems with cascaded erbium-doped fiber amplifiers (EDFA's). The difficulty in maintaining both a flattened gain and an adequate optical signal-to-noise ratio (SNR) for all channels arises from a nonuniform wavelengthdependent gain profile and saturation characteristics of each EDFA. The rapid accumulation of interchannel power variations and large differences in SNR among channels are undesirable. They may cause significant troubles to a system when power variations are beyond the receiver dynamic range and/or SNR's of some channels are too low to be detectable.

The problems associated with gain and/or SNR equalization recently have received considerable attentions. Several solutions have been proposed, such as the external gainequalization techniques [1]-[5], and the more useful intrinsic methods with optimal system variables [6], maintaining high inversion operations [7], [8], and optimal individual EDFA parameters [9], [10]. These intrinsic techniques, however, are effective only in the $1.55-\mu \mathrm{m}$ band, but can not operate properly in the whole $1530-1565 \mathrm{~nm}$ spectral range. In this work, we present a passive gain-equalization technique by sandwiching a section of a samarium-doped fiber (SDF) [11], [12] between two EDF segments to achieve a flattened gain over the whole EDFA spectral range. We fabricated gain fiber (i.e., EDF) and equalizer (i.e., SDF) using the modified chemical vapor deposition (MCVD) and solution soaking [13] techniques. Our experimental results demonstrate that this technique is suitable for multiwavelength wide-band WDM transmission systems containing EDFA cascades.

Manuscript received November 7, 1995; revised January 24, 1996.

$\mathrm{S}$.-K. Liaw is with the Telecommunication Laboratories, MOTC, and the Institute of Electro-Optical Engineering, National Chiao-Tung University, Taiwan, R.O.C.

Y.-K. Chen is with the Institute of Electro-Optical Engineering, National Sun Yat-Sen University, P.O. Box 59-63, Kaohsiung 80424, Taiwan, R.O.C Publisher Item Identifier S 1041-1135(96)05135.X.

\section{FABRICATION AND CHARACTERISTICS OF SM-FIBER}

The preform of SDF was fabricated by MCVD and solution soaking techniques. First, we deposited thirty cladding layers and four core layers in a silica quartz tube by passing appropriate reactant gases and dopants into the tube at about $1600{ }^{\circ} \mathrm{C}$. In the final core-deposition layer, we lowered down the torch temperature and increased $\mathrm{GeCl}_{4}$ and $\mathrm{SiCl}_{4}$ flows by two times to form a porous layer in order to catch the Smdopants in the subsequent solution soaking process. Secondly, we put the deposited quartz tube in the $\mathrm{SmCl}_{3}(0.5 \%$-Mole) and $\mathrm{Al}\left(\mathrm{NO}_{3}\right)_{3}$ solution for $60 \mathrm{~min}$ to obtain, by soaking technique, the required concentration of samarium ion. Finally, we dried and sintered the quartz tube by a collapse process to get the preform rod. The rod was drawn to form a singlemode SDF. The core/cladding diameter, numerical aperture, and cutoff wavelength are $5 / 125 \mu \mathrm{m}, 0.21$, and $1180 \mathrm{~nm}$, respectively.

The absorption spectrum of the SDF characterized by a set of bands in the near infrared region, is shown in Fig. 1(a). One could see that the SDF absorption is the band with a central wavelength of $1.525 \mu \mathrm{m}$ appears suitable for spectral filtering. The loss minimum occurs at $1.570 \mu \mathrm{m}$. The measured loss spectrum in $1525-1565 \mathrm{~nm}$ is shown in Fig. 1(b). It is clear that the splicing of an adequate length of SDF within EDFA's would be effective to make the gain spectrum flatten due to high-signal attenuation around the maximum amplification wavelength of $1.532 \mu \mathrm{m}$.

\section{EXPERIMENTS AND RESULTS}

The experimental setup is shown in Fig. 2. The gainequalized EDFA is a dual-pumped amplifier with a piece of SDF sandwiched between two EDF segments of the equal length. The alumino-germano-silicate EDF was also fabricated by the MCVD and solution soaking techniques. Each EDF is $4.5 \mathrm{~m}$ in length and $6 \mu \mathrm{m}$ in core diameter, and with cutoff wavelength of $1120 \mathrm{~nm}$ and a $5.6-\mathrm{dB} / \mathrm{m}$ peak absorption at $1532 \mathrm{~nm}$. Each 980-nm pumped laser diode has an output power of about $40 \mathrm{~mW}$. Two optical isolators, each with 45- $\mathrm{dB}$ isolation and 1-dB insertion loss, were added at the input and output ports to prevent optical reflections. Four DFB lasers at $1529,1541,1549$, and $1559 \mathrm{~nm}$, respectively, combined through a sixteen-channel diffraction-grating-based wavelength multiplexer and fed to a spectrum-flattened variable optical attenuator, were launched into the EDFA. The average splicing loss of EDF and SDF is about $0.8 \mathrm{~dB}$. 


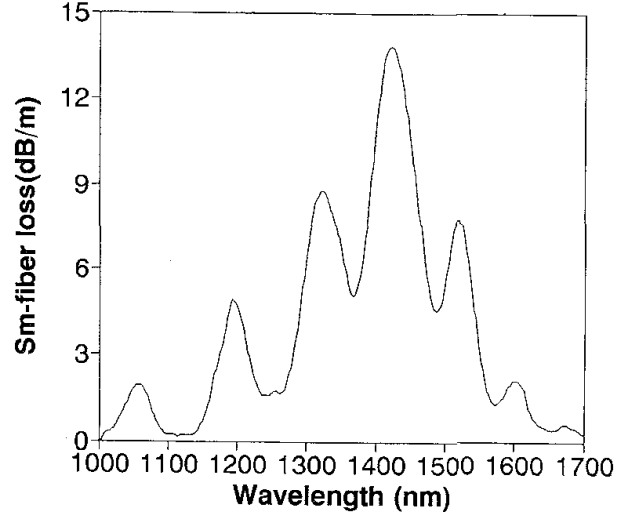

(a)

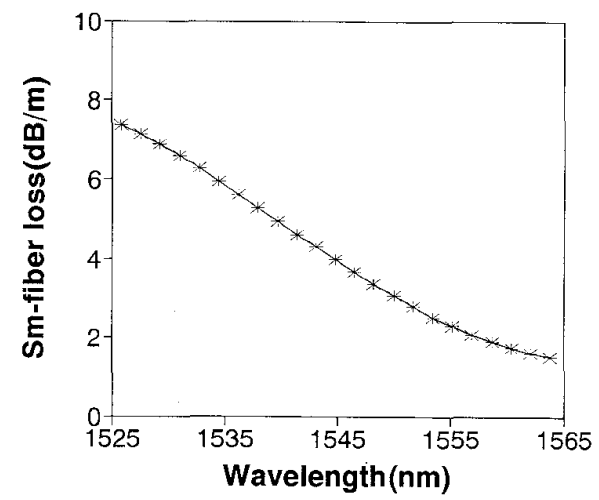

(b)

Fig. 1. Absorption spectrum of Sm-doped single-mode fiber in the wavelength range from (a) $1000-1700 \mathrm{~nm}$ and (b) $1525-1565 \mathrm{~nm}$.

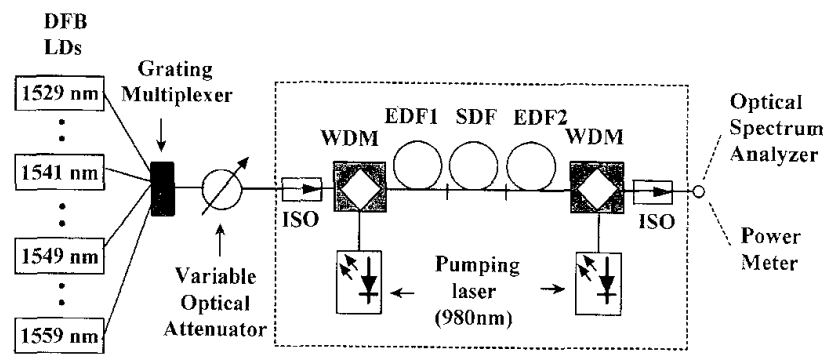

Fig. 2. Experimental setup of the Sm-fiber-equalized erbium-doped fiber amplifier. SDF: Sm-doped fiber. EDF: Erbium-doped fiber. ISO: Optical isolator. WDM: $0.98 / 1.55-\mu \mathrm{m}$ WDM coupler

Fig. 3 shows the fiber-to-fiber gain versus channel input power for an amplifier with different SDF lengths at 1529$\mathrm{nm}$ and $1559-\mathrm{nm}$ channels. For the equalizer with 2.4-m SDF, we find that the EDFA has the smallest difference in gain over a large channel input power ranging from -40 to -5 $\mathrm{dBm}$. For the input power level at $-15 \mathrm{dBm}$, the difference in gain between the $1529-\mathrm{nm}$ and $1559-\mathrm{nm}$ channels is less than $0.5 \mathrm{~dB}$. On the contrary, the EDFA without SDF (i.e., the $0.0-\mathrm{m}$ case) has the worst gain differential performance of about $6 \mathrm{~dB}$ and up to $14 \mathrm{~dB}$ for the input powers at $-15 \mathrm{dBm}$ and $-40 \mathrm{dBm}$, respectively. For the equalizer with SDF of

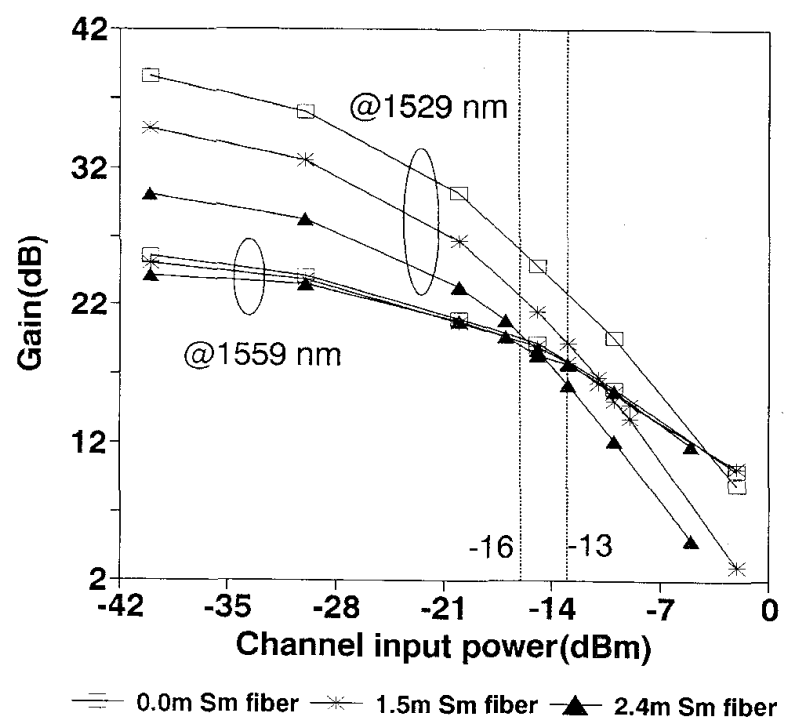

Fig. 3. Measured fiber-to-fiber gain versus channel input powers for amplifiers with different Sm-fiber lengths at $1529 \mathrm{~nm}$ and $1559 \mathrm{~nm}$.

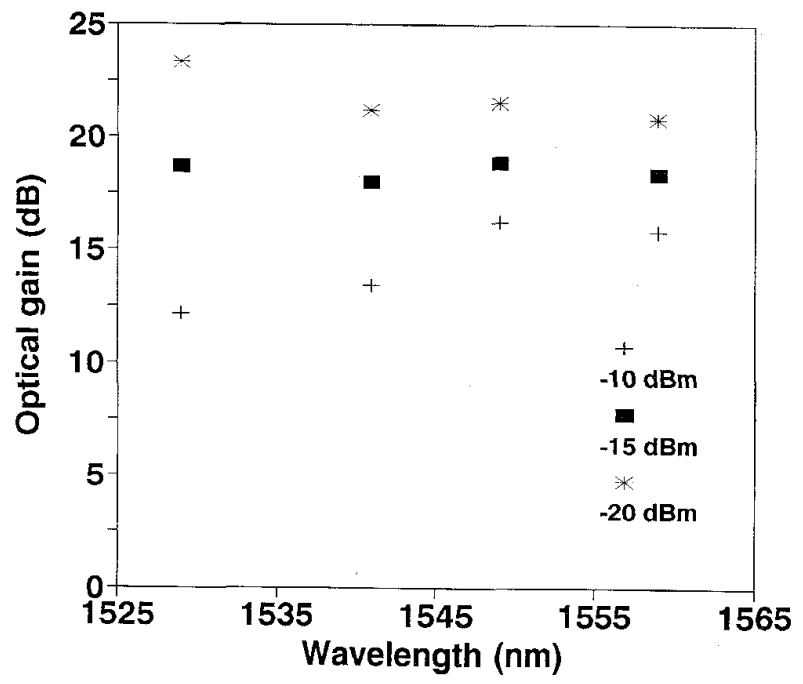

Fig. 4. Measured fiber-to-fiber gain for the equalized EDFA with 2.4-m Sm-fiber versus channel wavelengths for different input powers.

longer than $2.4 \mathrm{~m}$, the gain-equalization performance degrades, owing to the excessive absorption loss of the lengthened SDF. Fig. 4 shows the measured gain for the equalized EDFA with 2.4-m SDF versus channel wavelengths for different input powers. Note that a flattened wide-band gain spectrum and small inter-channel gain variations are achieved at the input power of $-15 \mathrm{dBm}$ than that of other input power levels of $-10,-13,-17$, and $-20 \mathrm{dBm}$. The averaged channel SNR is about $29 \mathrm{~dB}$. The measured noise figure of the equalized EDFA is less than $6.9,5.8,5.4$, and $5.6 \mathrm{~dB}$ at 1529,1541 , 1549 , and $1559 \mathrm{~nm}$, respectively, for an input signal level less than $-10 \mathrm{dBm}$. By lengthening the first section of EDF and maintaining high inversion operations to provide high gain and reduced the splicing loss of EDF and SDF, the characteristics of noise figure can be further improved. In consequence, the 
above experimental results have demonstrated the feasibility of the present passive gain-equalization method. In addition, this equalized EDFA is suitable for the application as an inline amplifier with the input power levels ranging from -16 to $-13 \mathrm{dBm}$, as the region between two vertical dot lines depicted in Fig. 3.

\section{CONCLUSION}

We have demonstrated a gain-equalized wide-band EDFA employing a sandwiched SDF. We have fabricated both the EDF and SDF by MCVD and solution soaking techniques. The experimental results have shown the capability of this equalized technique for multiwavelength wide-band WDM transmission systems containing concatenated chain of EDFA's.

\section{ACKNOWLEDGMENT}

The authors would like to thank H. Y. Yang, C. L. Tzeng, J. W. Liaw, and Y. K. Tu of the Taiwan Telecommunication Labs for their support. The authors are also indebted to Prof. S. M. Tseng for fruitful suggestions.

\section{REFERENCES}

[1] K. Inoue, T. Kominato, and H. Toba, "Tunable gain equalization using a Mach-Zehnder optical filter in multistage fiber amplifiers," IEEE Photon. Technol. Lett., vol. 3, no. 8, pp. 718-720, 1991.

[2] S. F. Su, R. Olshansky, G. Joyce, and J. E. Baran, "Gain equalization in multiwavelength lightwave systems using acoustooptic tunable filters," IEEE Photon. Technol. Lett., vol. 4, no. 3, pp. 269-271, 1992.

[3] A. R. Chraplyvy. J. A. Nagal, and R. W. Tkach, "Equalization in amplified WDM lightwave transmission systems," IEEE Photon. Technol. Lett., vol. 4, no. 8, pp. 920-922, 1992.
[4] A. F. Elrefaie, E. L. Goldstein, and S. Zaidi, "Fiber-amplifier cascades with gain equalization in multiwavelength unidirectional inter-office ring networks," IEEE Photon. Technol. Lett., vol. 5, no. 9, pp. 1026-1028, 1992.

[5] L. Eskildsen, E. Goldstein, V. da Silva, M. Andrejco, and Y. Silberberg, "Optical power equalization for multiwavelength fiber-amplifier cascades using periodic inhomogeneous broadening," IEEE Photon. Technol. Lett., vol. 5, no. 10, pp. 1118-1190, 1993.

[6] S. M. Hwang and A. E. Willner, "Guides for optimizing system performance for 20 WDM channels propagating through a cascade of EDFA's," IEEE Photon. Technol. Lett., vol. 5, no. 10, pp. 1190-1193, 1993.

[7] E. L. Goldstein, L. Eskidsen, C. Lin, and R. E. Tench, "Multiwavelength propagation in lightwave systems with strongly inverted fiber amplifiers," IEEE Photon. Technol. Lett., vol. 6, no. 2, pp. 266-269, 1994.

[8] R. G. Smart, J. W. Sulhoff, J. L. Zyskind, J. A. Nagel, and D. J. DiGiovanni, "Gain peaking in a chain of 980-nm-pumped erbium-doped fiber amplifiers," IEEE Photon. Technol. Lett., vol. 6, no. 3, pp. 380-382, 1994.

[9] M. A. Ali, A. F. Elrefaie, R. E. Wagner, F. Mendez, J. Pan, and S. A. Ahmed, "Optimized performance of erbium-doped fiber amplifiers in multiwavelength lightwave systems," IEEE Photon. Technol. Lett., vol. 6, no. 8, pp. 1039-1042, 1994.

[10] M. A. Ali, A. F. Elrefaie, R. E. Wagner, and S. A. Ahmed, "Performance of erbium-doped fiber amplifier cascades in WDM multiple access lightwave networks," IEEE Photon. Technol. Lett., vol. 6, no. 9, pp. $1142-1145,1994$.

[11] B. J. Ainslie, S. P. Craig, and S. T. Davey, "The absorption and fluorescence spectra of rare ions in silica-based monomode fiber," $J$. Lightwave Technol., vol. 6, no. 2, pp. 287-293, 1988.

[12] A. V. Belov, G. G. Deviatykh, E. M. Dianov, A. N. Guryanov, D. D. Gusovskiy, V. F. Khopin, and A. S. Kurkov, " $\mathrm{Sm}^{3+}$-doped fiber application to the spectral filtration in the range of $1530-1570 \mathrm{~nm}$," Sov. Lightwave Commun., vol. 2, pp. 265-268, 1992.

[13] J. E. Townsend, S. B. Poole, and D. N. Payne, "Solution doping technique for the fabrication of rare-earth doped optical fibers," Electron. Lett., vol. 23, pp. 329-331, 1987. 